\title{
Short-term changes in the belt/zone structure of Saturn's Southern Hemisphere (1996-2004)
}

\author{
S. Pérez-Hoyos ${ }^{1}$, A. Sánchez-Lavega ${ }^{1}$, and R. G. French ${ }^{2}$ \\ ${ }^{1}$ Departamento de Física Aplicada I, ETS Ingeniería, Universidad del País Vasco, Alameda deUrquijo s/n, 48013 Bilbao, Spain \\ e-mail: wubpehos@ibi.ehu.es \\ 2 Department of Astronomy, Wellesley College, Massachusetts, USA
}

Received 5 July 2006 / Accepted 8 September 2006

ABSTRACT

\begin{abstract}
Aims. The main goal of this paper is to study the reflectivity changes that affect Saturn's global belt/zone pattern on time-scales from weeks to a few months, and to determine their frequency and intensity, as well as the variations of the vertical cloud structure that cause such changes.

Methods. We have used 177 images from Hubble Space Telescope observations covering the 336-814 nm spectral range taken between 1996 and 2001, and the 255-890 nm range for observations from 2002 to 2004. These HST images were photometrically calibrated and geometrically corrected in order to quantify real reflectivity changes. We also used radiative transfer codes to retrieve vertical cloud structure models able to account for the observed changes, fitting the variation of absolute reflectivity from the center of the disk to the limb at the selected latitude at all wavelengths simultaneously.

Results. The results show that wavelength-dependent reflectivity changes are not in general related to localized disturbances (larger than the HST resolution of about $260 \mathrm{~km} /$ pixel), affecting instead an entire latitude band simultaneously. Those changes are most frequently found at equatorial and polar latitudes, brightening or darkening by $5 \%$ to $10 \%$ a latitude band typically less than $3^{\circ}$ wide. We find that reflectivity changes can be explained as a variation of the single scattering albedo of the tropospheric particles.

Conclusions. Our results confirm the kind of rapid changes observed during the Voyager mission in the belt/zone pattern of Saturn. They occur in narrow latitude bands due to a change in the cloud particle properties. This provides a framework for Cassini spacecraft observations, which will give the temporal and spatial resolution needed to understand Saturn cloud variability.
\end{abstract}

Key words. planets and satellites: general - planets and satellites: individual: Saturn - radiative transfer

\section{Introduction}

Saturn's appearance is organized in bright zones and dark belts, similarly to Jupiter. This banded aspect is not the same, however, in all spectral ranges, due to the wavelength-dependent processes affecting light scattering and absorption in the atmosphere (gas and hazes). Long-term changes (of the order of years) in the banded pattern or at hemispheric scale have been reported from ground-based observations (Sánchez-Lavega \& Battaner 1986; Sánchez-Lavega et al. 1993) and Hubble Space Telescope observations (Pérez-Hoyos et al. 2005; Karkoschka \& Tomasko 2005). Some of those changes are apparently related to the seasonal insolation cycle, enhanced by ring-shadowing at equatorial latitudes and Saturn's oblateness (Barnet et al. 1992). Current modeling suggests that they mainly affect the upper stratospheric haze layer (Pérez-Hoyos et al. 2005; Karkoschka \& Tomasko 2005), permanently present in Saturn atmosphere.

However, short-term changes (of the orders of weeks to a few months) of a particular belt or zone have not been well studied, although they were noted during the few months separating the Voyager 1 and 2 flybys (Smith et al. 1982). It is tempting to assign such changes to fast dynamical processes related to a localized disturbed area, as occurs for example in some belts of Jupiter (namely the Southern Equatorial Belt SEB, Sánchez-Lavega et al. 1996, and the North Temperate Belt NTB, Sánchez-Lavega et al. 1991; Rogers 1995). But the reason for these changes, their frequency and areas of activity are at present unknown, with the sole exception of the major Saturn band changes related to the outbursts known as Great White Spots that have been observed at several latitudes (Sánchez-Lavega 1982; Sánchez-Lavega et al. 1991, 1993).

The aim of this paper is therefore to address this issue, quantifying the frequency, wavelength dependence and latitude location (among other aspects) using a seven and a half-year survey with the Hubble Space Telescope. We will also analyze how these changes can be explained in terms of current vertical cloud structure models of Saturn's atmosphere.

In the next section we will review our Hubble Space Telescope observations to be analyzed in Sect. 3 in terms of the belt/zone pattern changes. Results based on vertical cloud structure modeling are given in Sect. 4 , before the main conclusions of this work are presented.

\section{Observations}

Hubble Space Telescope images of Saturn used in this work are presented in part in Cuzzi et al. (2002) and in Pérez-Hoyos et al. (2005). The long-term evolution of the atmosphere was studied in Pérez-Hoyos et al. (2005), and in this work we analyze observations in the same yearly campaign but over intervals of days, weeks and months.

Observational runs are presented in Table 1, resulting in a total of 177 images. These include images obtained in the five wide filters covering the $336-814 \mathrm{~nm}$ range, in the deep methane filter 
Table 1. HST observations.

\begin{tabular}{|c|c|c|}
\hline $\begin{array}{c}\text { Yearly } \\
\text { campaign }\end{array}$ & $\overline{\text { Date }}$ & Filters \\
\hline 1996-97 & $\begin{array}{l}09 / 30 / 1996 \\
10 / 14 / 1996 \\
01 / 10 / 1997\end{array}$ & $\begin{array}{c}F 336 W, F 439 W, F 555 W \\
F 675 W, F 814 W\end{array}$ \\
\hline 1997-98 & $\begin{array}{l}09 / 22 / 1997 \\
10 / 01 / 1997 \\
10 / 06 / 1997 \\
10 / 10 / 1997 \\
01 / 01 / 1998\end{array}$ & $\begin{array}{c}F 336 W, F 439 W, F 555 W \\
F 675 W, F 814 W\end{array}$ \\
\hline 1998-99 & $\begin{array}{l}07 / 28 / 1998 \\
10 / 13 / 1998 \\
10 / 18 / 1998 \\
10 / 24 / 1998\end{array}$ & $\begin{array}{c}F 336 W, F 439 W, F 555 W \\
F 675 W, F 814 W\end{array}$ \\
\hline 1999-2000 & $\begin{array}{l}08 / 25 / 1999 \\
09 / 03 / 1999 \\
09 / 07 / 1999\end{array}$ & $\begin{array}{c}F 336 W, F 439 W, F 555 W \\
F 675 W, F 814 W\end{array}$ \\
\hline $2000-01$ & $\begin{array}{l}08 / 04 / 2000 \\
11 / 20 / 2000 \\
11 / 24 / 2000 \\
12 / 06 / 2000\end{array}$ & $\begin{array}{c}F 336 W, F 439 W, F 555 W \\
F 675 W, F 814 W\end{array}$ \\
\hline 2001-02 & $\begin{array}{l}09 / 08 / 2001 \\
09 / 28 / 2001 \\
01 / 31 / 2002\end{array}$ & $\begin{array}{c}F 336 W, F 439 W, F 555 W \\
F 675 W, F 814 W\end{array}$ \\
\hline $2002-03$ & $\begin{array}{l}09 / 21 / 2002 \\
09 / 30 / 2002 \\
12 / 09 / 2002 \\
12 / 14 / 2002 \\
12 / 16 / 2002 \\
12 / 17 / 2002\end{array}$ & $\begin{array}{c}F 255 W, F 336 W, F 439 W \\
F 555 W, F 675 W, F 814 W \\
\text { FQCH4N }\end{array}$ \\
\hline 2003-04 & $\begin{array}{c}08 / 25 / 2003 \\
12 / 05 / 2003 \\
12 / 31 / 2003 \\
01 / 01 / 2004 \\
05 / 01 / 2004 \\
03 / 29 / 2004\end{array}$ & $\begin{array}{c}F 255 W, F 336 W, F 439 W \\
F 555 W, F 675 W, F 814 W \\
\text { FQCH4N }\end{array}$ \\
\hline
\end{tabular}

at $890 \mathrm{~nm}(\mathrm{FQCH} 4 \mathrm{~N})$ for the last two campaigns, and occasionally in the ultraviolet filter at $255 \mathrm{~nm}$. The time interval between observations varies from just one day to about three months, always within the same yearly campaign.

All of these images were navigated and photometrically calibrated as described in Pérez-Hoyos et al. (2005) and references therein. In order to eliminate reflectivity variations due to the various geometrical configurations, we performed a Minnaert fit (Minnaert 1941) to the absolute reflectivity data. However, some previous authors (Karkoschka \& Tomasko 1992, 1993) noted that this kind of geometrical correction does not take into account any dependence with the phase angle as presented by Lumme \& Irvine (1976). Although small (since Saturn's phase angle seen from Earth varies from $0^{\circ}$ to $6^{\circ}$ ), this effect is detectable in our data when comparing images taken at different phase angles. Therefore, we apply a complete geometrical Minnaert correction written in the form:

$(I / F)=(I / F)_{0}(\alpha) \mu_{0}^{k} \mu^{k-1}$

where the reflectivity $(I / F)$ is given in terms of the phase-angle $(\alpha)$ dependent absolute reflectivity $(I / F)_{0}(\alpha)$ and

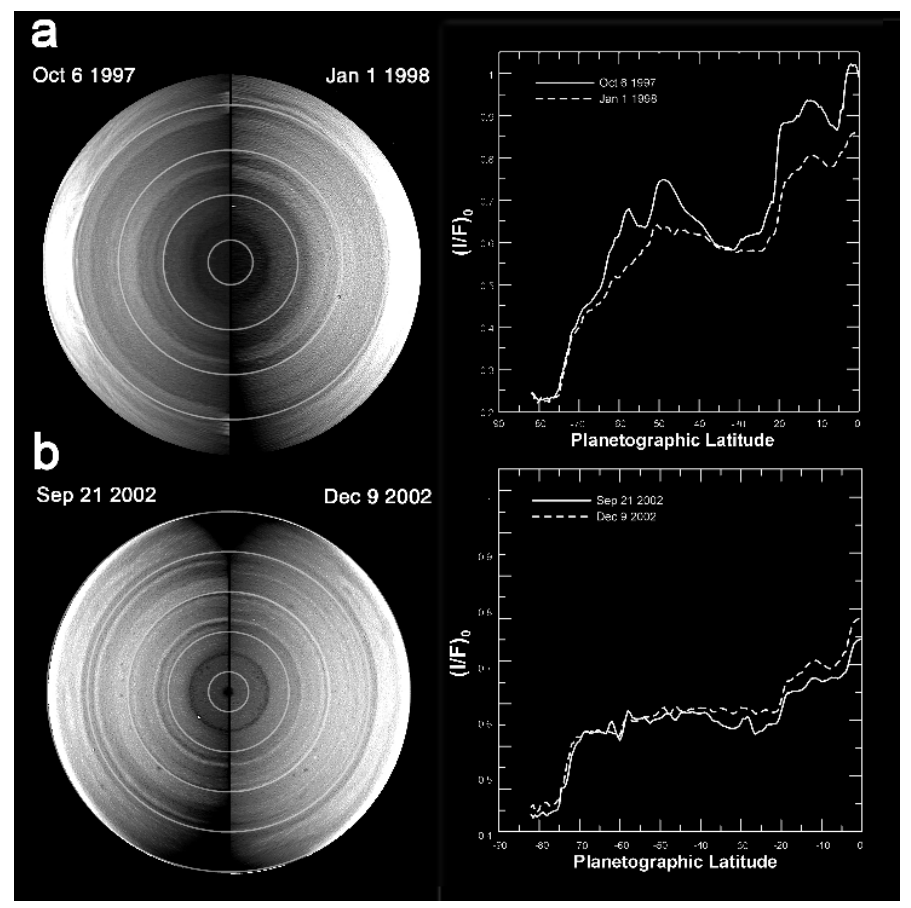

Fig. 1. South polar projections at $814 \mathrm{~nm}$ for a) the $1997-98$ and b) the 2002-03 campaigns over a 2-3 month interval in each case, showing representative changes in the belt/zone structure. The circles are spaced $20^{\circ}$ in latitude from $0^{\circ}$ to $-80^{\circ}$. The right panels show absolute reflectivity $(I / F)_{0}$ after correcting for solar phase variations using a Minnaert fit.

limb-darkening coefficient $k$, using the cosines of the angles of sunlight incidence and observation $\left(\mu_{0}, \mu\right)$ as independent variables.

Lumme \& Irvine (1976) found a phase-dependent reflectivity variation of 0.013 mag per degree of phase angle for Saturn, a value also used by Karkoschka \& Tomasko (1992, 1993). Since this effect should depend on the vertical cloud structure, and this is known to change with time (e.g. Pérez-Hoyos et al. 2005), we performed a linear fit of the hemispherically averaged brightness as a function of phase angle for each filter and year. This gave a value of $0.012 \pm 0.001 \mathrm{mag} / \mathrm{deg}$ (from $1 \%$ to $6 \%$ changes between observations) for the filters $F 439 W, F 555 W, F 675 W$ and $F 814 W, 0.008 \pm 0.001$ for $F 255 W$ (up to a $4 \%$ difference) and no appreciable variation for $F 336 W$ and FQCH4N.

This allowed us to extract the center to limb variations of the absolute reflectivity every $0.5^{\circ}$ in latitude and then fit each scan to a Minnaert law, giving finally the dependence of the Minnaert parameters $k$ and $(I / F)_{0}$ with latitude with half a degree precision. As an example, Fig. 1 displays polar projection together with the corrected $(I / F)_{0}$ for two different years in the filter centered at $814 \mathrm{~nm}$. The absolute reflectivity changes were much more conspicuous in 1997-98 than in 2002. Figure 2 shows the calibrated and geometrically-corrected results for the 2003-04 observations. Similar results are found for each yearly campaign.

With respect to the error bars in $(I / F)$, Cuzzi et al. (2002) gave a random error of about $\sigma=1 \%$. Taking into account the additional data reductions described above, we assume the random error in $(I / F)_{0}$ to be $\sigma=1-2 \%$, which seems quite reasonable in view of the actual dispersion of the data. 

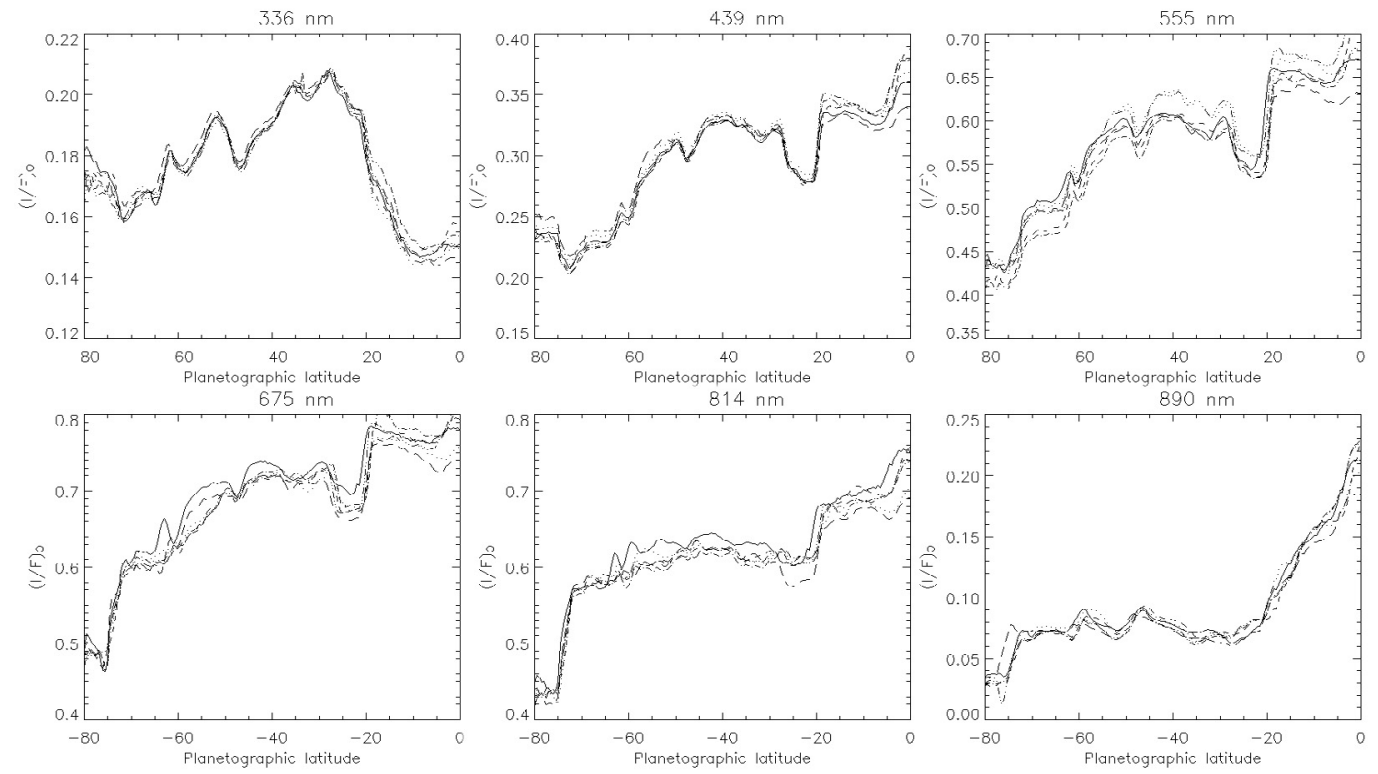

Fig. 2. Absolute reflectivity $(I / F)_{0}$ as a function of latitude for the 2003-04 campaign in all available filters, except $F 255 W$, corrected for phase angle variations. Each line represents one observation date, as given in Table 1.
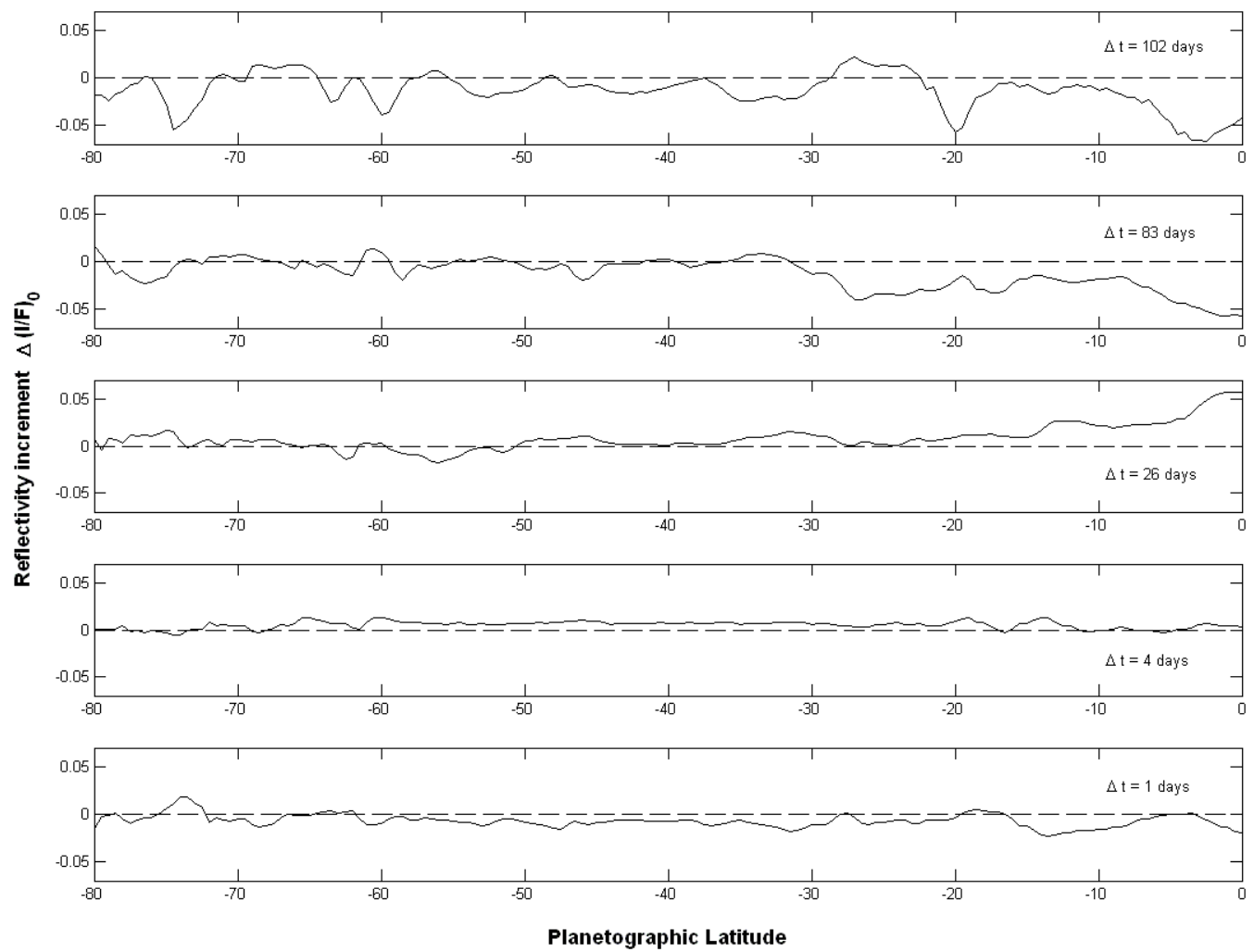

Fig. 3. Differences in the corrected absolute reflectivity $(I / F)_{0}$ on consecutive dates for observations of the 2003-04 yearly campaign in the filter centered at $814 \mathrm{~nm}$. The time interval $\Delta t$ between one image and the following is also shown. Dashed lines show the expected behavior in the absence of any reflectivity changes $\left(\Delta(I / F)_{0}=0\right)$.

\section{Statistics of the belt/zone changes}

There are several observable parameters that can be affected by changes of the belt/zone structure over a given range of latitudes: (1) Intensity (reflectivity) variations, especially when compared to neighboring quiescent regions, are most easily detected, given an accurate photometric reduction process and geometrical correction algorithm. We focus on these in this work. (2) Changes in the limb darkening behavior of the belts and zones are in principle detectable with a similar technique, but for our observations, these had relatively large uncertainties, and it probably requires better temporal sampling to identify such changes convincingly. (3) The location and width of belts and zones can change with time. To determine changes in the position of the 
belts and zones, we calculated the latitudinal reflectivity gradient $\partial(I / F)_{0} / \partial \varphi$, where $\varphi$ is the planetographic latitude. Such variations are also revealed by computing reflectivity increments or decrements at fixed latitudes as a function of time, as we did. Thus, to determine the regions in which reflectivity changes are located, we calculated relative changes from one date to the following at each latitude. Only those regions with variations greater than $3 \sigma$, where $\sigma$ is the random error in reflectivity, were considered. Taking into account the different number of images obtained each year, we found comparable changes over all years, although somewhat higher in 1998.

In a similar way, we compared the relative frequency of changes at each filter. The most frequent changes turned out to be in the $F 255 \mathrm{~W}$ and FQCH4N filters, both of which sense mainly the stratospheric haze and the upper level of the tropospheric haze (Pérez-Hoyos \& Sánchez-Lavega 2006). Since these are the less common filters in this study, we cannot say much more about this issue.

In general terms, between a Saturn image in a given filter and the following in the same yearly campaign, we observed on the order of 5 to 10 regions of change, using the criterion defined above. However, this depends on the time elapsed between one observation and the following. Figure 3 displays the reflectivity variations derived from comparison images at $814 \mathrm{~nm}$ in the 2003-04 campaign on consecutive dates. Typically, it takes about five days for a detectable variation in the belt/zone pattern to develop. Convincing evidence of shorter term time variations (for example, over a single day) requires careful examination of the corresponding images.

Our statistical survey of observed zonal reflectivity changes is summarized in Fig. 4. The latitude distribution of changes is shown in the panel 4a. There are two main wide regions of activity. The first is in the Equatorial Zone (between $0^{\circ}$ and $20^{\circ} \mathrm{S}$ ) and the second is southward of $60^{\circ} \mathrm{S}$. Close to the equator we can also distinguish between two different kinds of disturbances. The first is related to isolated large scale atmospheric features observed at those latitudes (Sánchez-Lavega et al. 1999, 2004), whereas the second is located close to the reflectivity gradient at about $20^{\circ} \mathrm{S}$ whose location has been observed to change slightly with time. On the other hand, reflectivity changes were also very frequent in the polar region, southward of $60^{\circ} \mathrm{S}$. The consideration of a single relative error for all latitudes may overestimate the number of polar changes, since these regions are susceptible to more critical limb-fitting problems. Nevertheless, visual inspection of the images qualitatively confirms the presence of more frequent variations in the polar region.

With respect to the latitude width of the changes, Fig. $4 \mathrm{~b}$ shows that about $75 \%$ of them span a width of $3^{\circ}$ or less. About $80 \%$ of the observed phenomena produce a reflectivity variation less than $20 \%$ of the initial value. Brightening seems to be slightly more common than darkening, although both phenomena are pretty similar.

Perhaps the most interesting aspect of the non-equatorial albedo changes is that they were not preceded by a dynamical disturbance of the region (i.e. by the presence of an individual spot), at least at the HST spatial resolution ( $260 \mathrm{~km} / \mathrm{pixel})$, as happens, for example, on Jupiter or during the Great White Storms on Saturn (Sánchez-Lavega et al. 1991). No large-scale belt/zone pattern change was observed to be correlated with any individual feature. This implies that changes are truly circumferencial or, more likely, that atmospheric events that perturbate the latitude band are located deeper than our sounding levels.

In a similar way, observed atmospheric features had no effect in the belt/zone pattern. For example, our team reported
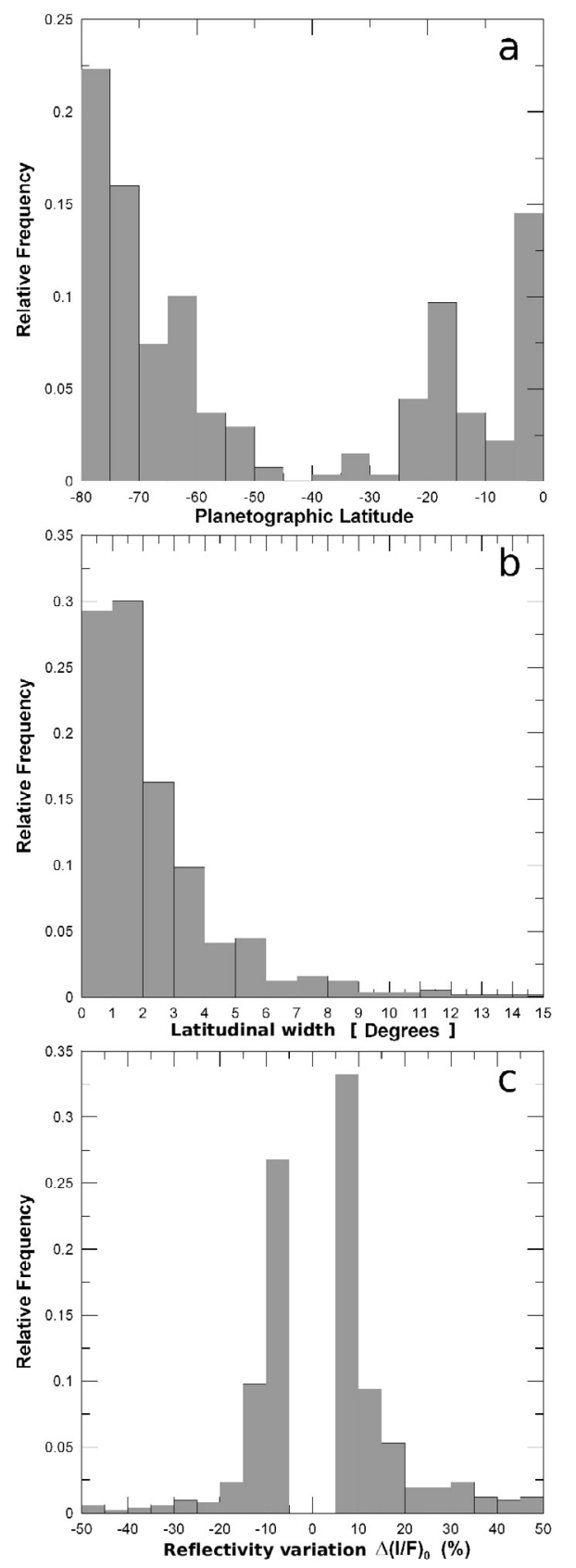

Fig. 4. Statistics of the observed changes in the belt/zone pattern. The upper panel shows the relative frequency of changes as a function of latitude, the middle panel shows the width (in degrees) of the bands affected by each change, and the last panel shows the a histogram of observed intensity changes.

(Sánchez-Lavega et al. 2004) the presence of convective storms (of the same type as the so-called "Dragon Storm" reported by the Cassini-ISS team in the Saturn's storm alley, Porco et al. 2005) at the mid-latitudes and other features that produced no appreciable variation of the latitude band at their location.

\section{Vertical cloud structure modeling}

In order to constrain the origin of the observed variations of absolute reflectivity, we performed calculations using vertical cloud structure models similar to those presented in Pérez-Hoyos et al. (2005) for the situations shown in Fig. 1 (1997-98) and Fig. 2 (2003-04). We took as a reference model that closest in 


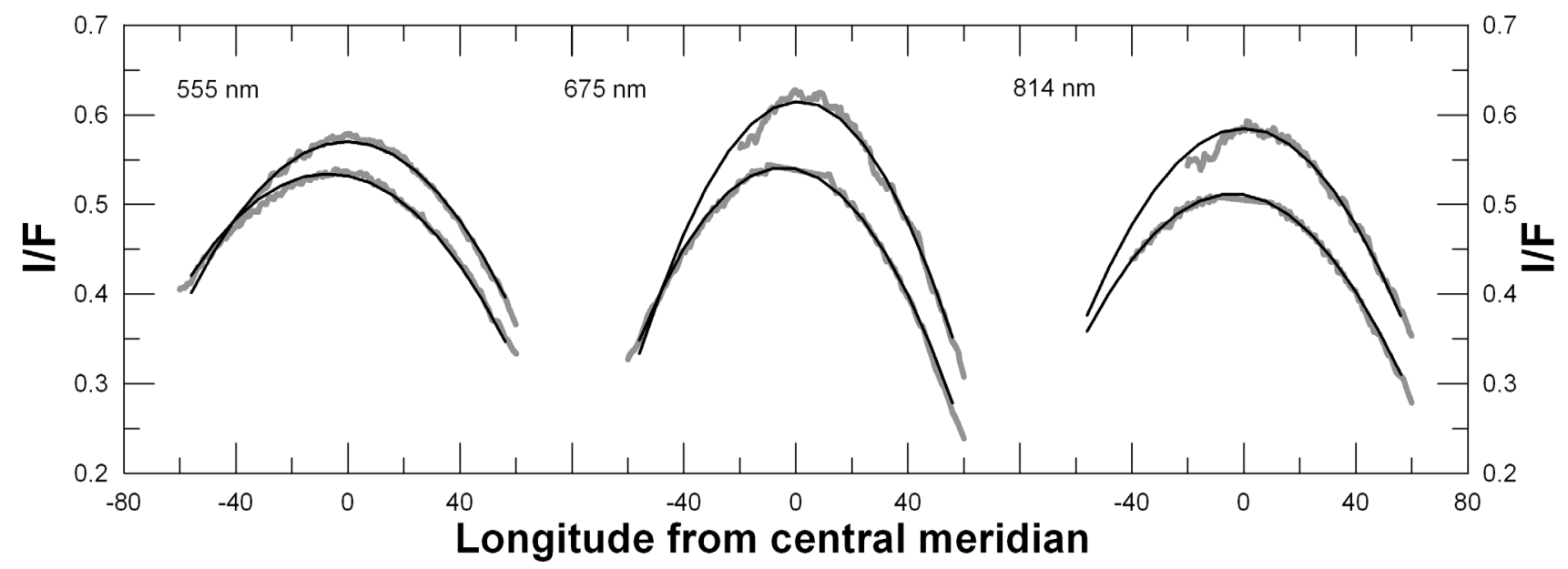

Fig. 5. Center to limb scans (grey lines) at $49^{\circ} \mathrm{S}$ for two different dates (10/10/1997, upper curves, and 01/01/1998, lower curves), and three wavelengths corresponding to the $F 555 \mathrm{~W}, F 675 \mathrm{~W}$ and $F 814 \mathrm{~W}$ filters. The black lines represent the best-fit cloud structure model for each scan.

Table 2. Variation of the tropospheric particles' single scattering albedo.

\begin{tabular}{ccc}
\hline \hline Filter & $\begin{array}{c}\varpi_{0} \\
(10 / 10 / 1997)\end{array}$ & $\begin{array}{c}\varpi_{0} \\
(01 / 01 / 1998)\end{array}$ \\
\hline$F 336 W$ & $0.70 \pm 0.01$ & $0.68 \pm 0.01$ \\
$F 439 W$ & $0.915 \pm 0.005$ & $0.891 \pm 0.005$ \\
$F 555 W$ & $0.984 \pm 0.001$ & $0.977 \pm 0.001$ \\
$F 675 W$ & $0.996 \pm 0.001$ & $0.972 \pm 0.001$ \\
$F 814 W$ & $0.999 \pm 0.001$ & $0.994 \pm 0.001$ \\
\hline
\end{tabular}

time from Pérez-Hoyos et al. (2005) and studied which parameters should be changed to account for the variations in the reflectivity and limb-darkening behavior.

For the 1997-98 observations, we studied the very large reflectivity change in the mid-latitudes shown in Fig. 1. Figure 5 shows our model fits compared to the observations of the center-to-limb reflectivity variation at three wavelengths, for 10 October 1997 and 1 January 1998.

The only parameter able to reproduce the observed darkening was the single scattering albedo of the tropospheric particles. We show in Table 2 the variations in the particle single scattering albedo with wavelength for the two dates. This kind of variation points to small changes in the particle size, as suggested by Karkoschka \& Tomasko (2005). Other latitudes analyzed in this 1997-98 yearly campaign yielded similar conclusions, although smaller temporal brightness changes sometimes resulted in variations of single scattering albedo that were comparable to the error bars.

We also analyzed data from the 2002-03 yearly campaign (covering the $255-890 \mathrm{~nm}$ spectral range) at equatorial, intermediate and polar latitudes. We found the same results as in the 1997-98 campaign: the single-scattering albedo variation alone is able to reproduce observed reflectivity changes.

\section{Conclusions}

Analysis of Saturn images in the $255-890 \mathrm{~nm}$ wavelength range shows changes with different time-scales (from days to months) in the belt/zone pattern. Those changes, in general, seem not to be preceded by any individual atmospheric disturbance localized in latitude or longitude, evolving instead gradually and simultaneously in the whole band. Modeling the reflectivity changes suggests variations in the single-scattering albedo of the particles forming the tropospheric haze (probably induced by small changes in the size or composition of the particles) as the most likely explanation for most of the observed variations. It remains to determine what kind of disturbance (dynamical, photochemical) could produce the reflectivit y changes in a matter of weeks. This issue is of great interest for the interpretation of Cassini spacecraft observations that will provide the time and spectral baseline needed to understand these processes in Saturn's atmosphere.

Acknowledgements. S.P.-H. and A.S.-L. were supported by the MCYT Plan Nacional de Astronomía y Astrofísica 2003-03216, fondos FEDER, and Grupos UPV 15946/2004. S.P.-H. acknowledges a Ph.D. fellowship from the Spanish MEC. The NASA/ESA Hubble Space Telescope is operated by the Association of Universities for Research in Astronomy under NASA contract NAS5-26555. RF acknowledges support from the NASA Planetary Geology and Geophysics Program.

\section{References}

Barnet, C. D., Beebe, R. F., \& Conrath, B. J. 1992, Icarus, 98, 94 Cuzzi, J. N., French, R. G., \& Dones, L. 2002, Icarus, 158, 199 Ingersoll, A. P. 1990, Science, 248, 308

Karkoschka, E., \& Tomasko, M. G. 1992, Icarus, 97, 161

Karkoschka, E., \& Tomasko, M. G. 1993, Icarus, 106, 428

Karkoschka, E., \& Tomasko, M. G. 2005, Icarus, 179, 195

Lumme, K., \& Irvine, W. M. 1976, AJ, 81, 865

Minnaert, M. 1941, ApJ, 93, 403

Pérez-Hoyos, S., Sánchez-Lavega, A., French, R. G., \& Rojas, J. F. 2005, Icarus, 176,155

Pérez-Hoyos, S., \& Sánchez-Lavega, A. 2006, Icarus, 180, 161

Porco, C. C., Baker, E., Barbara, J., et al. 2005, Science, 307, 1243

Rogers, J. H. 1995, The giant planet Jupiter (New York: Cambridge University Press), USA

Sánchez-Lavega, A. 1982, Icarus, 49, 1

Sánchez-Lavega, A., \& Battaner, E. 1986, A\&AS, 64, 287

Sánchez-Lavega, A., Colas, F., Lecacheux, J., Laques, P., Miyazaki, I., \& Parker, D. 1991, Nature, 353, 397

Sánchez-Lavega, A., Lecacheaux, J., Colas, F., \& Laques, P. 1993, J. Geophys. Res., 98, 18857

Sánchez-Lavega, A., Gómez, J. M., Lecacheux, J., et al. 1996, Icarus, 121, 18

Sánchez-Lavega, A., Lecacheux, J. Colas, F., Rojas, J. F., \& Gmez, J. M. 1999, Planet. Space Sci., 47, 1277, 1283

Sánchez-Lavega, A., Hueso, R., Pérez-Hoyos, S., Rojas, J. F., \& French, R. G. 2004, Icarus, 170, 519, 523

Smith, B. A., Soderblom, L., Batson, R. M., et al. 1982, Science, 215, 504 